\title{
LOWER BOUNDS FOR MAHLER MEASURE THAT DEPEND ON THE NUMBER OF MONOMIALS
}

\author{
SHABNAM AKHTARI AND JEFFREY D. VAALER
}

\begin{abstract}
We prove a new lower bound for the Mahler measure of a polynomial in one and in several variables that depends on the complex coefficients, and the number of monomials. In one variable our result generalizes a classical inequality of Mahler. In $M$ variables our result depends on $\mathbb{Z}^{M}$ as an ordered group, and in general our lower bound depends on the choice of ordering.
\end{abstract}

\section{INTRODUCTION}

Let $P(z)$ be a polynomial in $\mathbb{C}[z]$ that is not identically zero. We assume to begin with that $P$ has degree $N$, and that $P$ factors into linear factors in $\mathbb{C}[z]$ as

$$
P(z)=c_{0}+c_{1} z+c_{2} z^{2}+\cdots+c_{N} z^{N}=c_{N} \prod_{n=1}^{N}\left(z-\alpha_{n}\right) .
$$

If $e: \mathbb{R} / \mathbb{Z} \rightarrow \mathbb{T}$ is the continuous isomorphism given by $e(t)=e^{2 \pi i t}$, then the Mahler measure of $P$ is the positive real number

$$
\mathfrak{M}(P)=\exp \left(\int_{\mathbb{R} / \mathbb{Z}} \log |P(e(t))| \mathrm{d} t\right)=\left|c_{N}\right| \prod_{n=1}^{N} \max \left\{1,\left|\alpha_{n}\right|\right\} .
$$

The equality on the right of (1.2) follows from Jensen's formula. If $P_{1}(z)$ and $P_{2}(z)$ are both nonzero polynomials in $\mathbb{C}[z]$, then it is immediate from (1.2) that

$$
\mathfrak{M}\left(P_{1} P_{2}\right)=\mathfrak{M}\left(P_{1}\right) \mathfrak{M}\left(P_{2}\right) .
$$

Mahler measure plays an important role in number theory and in algebraic dynamics, as discussed in [6, 12, [14, Chapter 5], and [16. Here we restrict our attention to the problem of proving a lower bound for $\mathfrak{M}(P)$ when the polynomial $P(z)$ has complex coefficients. We establish an analogous result for polynomials in several variables.

For $P(z)$ of degree $N$ and given by (1.1), there is a well known lower bound due to Mahler which asserts that

$$
\left|c_{n}\right| \leq\left(\begin{array}{c}
N \\
n
\end{array}\right) \mathfrak{M}(P), \quad \text { for each } n=0,1,2, \ldots, N .
$$

The inequality (1.3) is implicit in [9, and is stated explicitly in [11, section 2], (see also the proof in [1, Theorem 1.6.7]). If

$$
P(z)=(z \pm 1)^{N},
$$

2010 Mathematics Subject Classification. 11R06.

Key words and phrases. Mahler Measure, polynomial inequalities.

Shabnam Akhtari's research is funded by the NSF grant DMS-1601837. 
then there is equality in (1.3) for each $n=0,1,2, \ldots, N$.

We now assume that $P(z)$ is a polynomial in $\mathbb{C}[z]$ that is not identically zero, and we assume that $P(z)$ is given by

$$
P(z)=c_{0} z^{m_{0}}+c_{1} z^{m_{1}}+c_{2} z^{m_{2}}+\cdots+c_{N} z^{m_{N}},
$$

where $N$ is a nonnegative integer, and $m_{0}, m_{1}, m_{2}, \ldots, m_{N}$, are nonnegative integers such that

$$
m_{0}<m_{1}<m_{2}<\cdots<m_{N} .
$$

We wish to establish a lower bound for $\mathfrak{M}(P)$ which depends on the coefficients and on the number of monomials, but which does not depend on the degree of $P$. Such a result was recently proved by Dobrowolski and Smyth [5. We use a similar argument, but we obtain a sharper result that includes Mahler's inequality (1.3) as a special case.

Theorem 1.1. Let $P(z)$ be a polynomial in $\mathbb{C}[z]$ that is not identically zero, and is given by (1.4). Then we have

$$
\left|c_{n}\right| \leq\left(\begin{array}{c}
N \\
n
\end{array}\right) \mathfrak{M}(P), \quad \text { for each } n=0,1,2, \ldots, N .
$$

Let $f: \mathbb{R} / \mathbb{Z} \rightarrow \mathbb{C}$ be a trigonometric polynomial, not identically zero, and a sum of at most $N+1$ distinct characters. Then we can write $f$ as

$$
f(t)=\sum_{n=0}^{N} c_{n} e\left(m_{n} t\right)
$$

where $c_{0}, c_{1}, c_{2}, \ldots, c_{N}$, are complex coefficients, and $m_{0}, m_{1}, m_{2}, \ldots, m_{N}$, are integers such that

$$
m_{0}<m_{1}<m_{2}<\cdots<m_{N} .
$$

As $f$ is not identically zero, the Mahler measure of $f$ is the positive number

$$
\mathfrak{M}(f)=\exp \left(\int_{\mathbb{R} / \mathbb{Z}} \log |f(t)| \mathrm{d} t\right) .
$$

It is trivial that $f(t)$ and $e\left(-m_{0} t\right) f(t)$ have the same Mahler measure. Thus we get the following alternative formulation of Theorem 1.1

Corollary 1.1. Let $f(t)$ be a trigonometric polynomial with complex coefficients that is not identically zero, and is given by (1.7). Then we have

$$
\left|c_{n}\right| \leq\left(\begin{array}{c}
N \\
n
\end{array}\right) \mathfrak{M}(f), \quad \text { for each } n=0,1,2, \ldots, N .
$$

For positive integers $M$ we will prove an extension of Corollary 1.1 to trigonometric polynomials

$$
F:(\mathbb{R} / \mathbb{Z})^{M} \rightarrow \mathbb{C},
$$

that are not identically zero. The Fourier transform of $F$ is the function

$$
\widehat{F}: \mathbb{Z}^{M} \rightarrow \mathbb{C}
$$

defined at each lattice point $\boldsymbol{k}$ in $\mathbb{Z}^{M}$ by

$$
\widehat{F}(\boldsymbol{k})=\int_{(\mathbb{R} / \mathbb{Z})^{M}} F(\boldsymbol{x}) e\left(-\boldsymbol{k}^{T} \boldsymbol{x}\right) \mathrm{d} \boldsymbol{x} .
$$


In the integral on the right of (1.10) we write $\mathrm{d} \boldsymbol{x}$ for integration with respect to a Haar measure on the Borel subsets of $(\mathbb{R} / \mathbb{Z})^{M}$ normalized so that $(\mathbb{R} / \mathbb{Z})^{M}$ has measure 1. We write $\boldsymbol{k}$ for a (column) vector in $\mathbb{Z}^{M}, \boldsymbol{k}^{T}$ for the transpose of $\boldsymbol{k}, \boldsymbol{x}$ for a (column) vector in $(\mathbb{R} / \mathbb{Z})^{M}$, and therefore

$$
\boldsymbol{k}^{T} \boldsymbol{x}=k_{1} x_{1}+k_{2} x_{2}+\cdots+k_{N} x_{N} .
$$

As $F$ is not identically zero, the Mahler measure of $F$ is the positive real number

$$
\mathfrak{M}(F)=\exp \left(\int_{(\mathbb{R} / \mathbb{Z})^{M}} \log |F(\boldsymbol{x})| \mathrm{d} \boldsymbol{x}\right) .
$$

We assume that $\mathfrak{S} \subseteq \mathbb{Z}^{M}$ is a nonempty, finite set that contains the support of $\widehat{F}$. That is, we assume that

$$
\left\{\boldsymbol{k} \in \mathbb{Z}^{M}: \widehat{F}(\boldsymbol{k}) \neq 0\right\} \subseteq \mathfrak{S},
$$

and therefore $F$ has the representation

$$
F(\boldsymbol{x})=\sum_{\boldsymbol{k} \in \mathfrak{S}} \widehat{F}(\boldsymbol{k}) e\left(\boldsymbol{k}^{T} \boldsymbol{x}\right) .
$$

Basic results in this setting can be found in Rudin [13, Sections 8.3 and 8.4].

If $\boldsymbol{\alpha}=\left(\alpha_{m}\right)$ is a (column) vector in $\mathbb{R}^{M}$, we write

$$
\varphi_{\boldsymbol{\alpha}}: \mathbb{Z}^{M} \rightarrow \mathbb{R}
$$

for the homomorphism given by

$$
\varphi_{\boldsymbol{\alpha}}(\boldsymbol{k})=\boldsymbol{k}^{T} \boldsymbol{\alpha}=k_{1} \alpha_{1}+k_{2} \alpha_{2}+\cdots+k_{M} \alpha_{M} .
$$

It is easy to verify that $\varphi_{\boldsymbol{\alpha}}$ is an injective homomorphism if and only if the coordinates $\alpha_{1}, \alpha_{2}, \ldots, \alpha_{M}$, are $\mathbb{Q}$-linearly independent real numbers.

Let the nonempty, finite set $\mathfrak{S} \subseteq \mathbb{Z}^{M}$ have cardinality $N+1$, where $0 \leq N$. If $\varphi_{\boldsymbol{\alpha}}$ is an injective homomorphism, then the set

$$
\left\{\varphi_{\boldsymbol{\alpha}}(\boldsymbol{k}): \boldsymbol{k} \in \mathfrak{S}\right\}
$$

consists of exactly $N+1$ real numbers. It follows that the set $\mathfrak{S}$ can be indexed so that

$$
\mathfrak{S}=\left\{\boldsymbol{k}_{0}, \boldsymbol{k}_{1}, \boldsymbol{k}_{2}, \ldots, \boldsymbol{k}_{N}\right\}
$$

and

$$
\varphi_{\boldsymbol{\alpha}}\left(\boldsymbol{k}_{0}\right)<\varphi_{\boldsymbol{\alpha}}\left(\boldsymbol{k}_{1}\right)<\varphi_{\boldsymbol{\alpha}}\left(\boldsymbol{k}_{2}\right)<\cdots<\varphi_{\boldsymbol{\alpha}}\left(\boldsymbol{k}_{N}\right) .
$$

By using a limiting argument introduced in a paper of Boyd [2, we will prove the following generalization of (1.8).

Theorem 1.2. Let $F:(\mathbb{R} / \mathbb{Z})^{M} \rightarrow \mathbb{C}$ be a trigonometric polynomial that is not identically zero, and is given by (1.12). Let $\varphi_{\boldsymbol{\alpha}}: \mathbb{Z}^{M} \rightarrow \mathbb{R}$ be an injective homomorphism, and assume that the finite set $\mathfrak{S}$, which contains the support of $\widehat{F}$, is indexed so that (1.14) and (1.15) hold. Then we have

$$
\left|\widehat{F}\left(\boldsymbol{k}_{n}\right)\right| \leq\left(\begin{array}{c}
N \\
n
\end{array}\right) \mathfrak{M}(F), \quad \text { for each } n=0,1,2, \ldots, N .
$$


Let $F$ and $\varphi_{\boldsymbol{\alpha}}: \mathbb{Z}^{M} \rightarrow \mathbb{R}$ be as in the statement of Theorem [1.2 and then let $\varphi_{\boldsymbol{\beta}}: \mathbb{Z}^{M} \rightarrow \mathbb{R}$ be a second injective homomorphism. It follows that $\mathfrak{S}$ can be indexed so that (1.14) and (1.15) hold, and $\mathfrak{S}$ can also be indexed so that

$$
\mathfrak{S}=\left\{\ell_{0}, \ell_{1}, \ell_{2}, \ldots, \ell_{N}\right\}
$$

and

$$
\varphi_{\boldsymbol{\beta}}\left(\ell_{0}\right)<\varphi_{\boldsymbol{\beta}}\left(\ell_{1}\right)<\varphi_{\boldsymbol{\beta}}\left(\ell_{2}\right)<\cdots<\varphi_{\boldsymbol{\beta}}\left(\ell_{N}\right) .
$$

In general the indexing (1.14) is distinct from the indexing (1.17). Therefore the system of inequalities

$$
\left|\widehat{F}\left(\boldsymbol{k}_{n}\right)\right| \leq\left(\begin{array}{c}
N \\
n
\end{array}\right) \mathfrak{M}(F), \quad \text { for each } n=0,1,2, \ldots, N,
$$

and

$$
\left|\widehat{F}\left(\ell_{n}\right)\right| \leq\left(\begin{array}{c}
N \\
n
\end{array}\right) \mathfrak{M}(F), \quad \text { for each } n=0,1,2, \ldots, N,
$$

which follow from Theorem 1.2, are different, and in general neither system of inequalities implies the other.

\section{Proof of Theorem 1.1}

It follows from (1.2) that the polynomial $P(z)$, and the polynomial $z^{-m_{0}} P(z)$, have the same Mahler measure. Hence we may assume without loss of generality that the exponents $m_{0}, m_{1}, m_{2}, \ldots, m_{N}$, in the representation (1.4) satisfy the more restrictive condition

$$
0=m_{0}<m_{1}<m_{2}<\cdots<m_{N} .
$$

If $N=0$ then (1.6) is trivial. If $N=1$, then

$$
\left(\begin{array}{l}
1 \\
0
\end{array}\right)=\left(\begin{array}{l}
1 \\
1
\end{array}\right)=1
$$

and using Jensen's formula we find that

$$
\mathfrak{M}\left(c_{0}+c_{1} z^{m_{1}}\right)=\max \left\{\left|c_{0}\right|,\left|c_{1}\right|\right\} .
$$

Therefore the inequality (1.6) holds if $N=1$. Throughout the remainder of the proof we assume that $2 \leq N$, and we argue by induction on $N$. Thus we assume that the inequality (1.6) holds for polynomials that can be expressed as a sum of strictly less than $N+1$ monomials.

Besides the polynomial

$$
P(z)=c_{0} z^{m_{0}}+c_{1} z^{m_{1}}+c_{2} z^{m_{2}}+\cdots+c_{N} z^{m_{N}},
$$

we will work with the polynomial

$$
Q(z)=z^{m_{N}} P\left(z^{-1}\right)=c_{0} z^{m_{N}-m_{0}}+c_{1} z^{m_{N}-m_{1}}+c_{2} z^{m_{N}-m_{2}}+\cdots+c_{N} .
$$

It follows from (1.2) that

$$
\mathfrak{M}(Q)=\exp \left(\int_{\mathbb{R} / \mathbb{Z}} \log \left|e\left(m_{N} t\right) P(e(-t))\right| \mathrm{d} t\right)=\mathfrak{M}(P) .
$$

Next we apply an inequality of Mahler [10] to conclude that both

$$
\mathfrak{M}\left(P^{\prime}\right) \leq m_{N} \mathfrak{M}(P), \quad \text { and } \quad \mathfrak{M}\left(Q^{\prime}\right) \leq m_{N} \mathfrak{M}(Q)
$$


Because

$$
P^{\prime}(z)=\sum_{n=1}^{N} c_{n} m_{n} z^{m_{n}-1}
$$

is a sum of strictly less than $N+1$ monomials, we can apply the inductive hypothesis to $P^{\prime}$. It follows that

$$
\left|c_{n}\right| m_{n} \leq\left(\begin{array}{c}
N-1 \\
n-1
\end{array}\right) \mathfrak{M}\left(P^{\prime}\right) \leq m_{N}\left(\begin{array}{c}
N-1 \\
n-1
\end{array}\right) \mathfrak{M}(P)
$$

for each $n=1,2, \ldots, N$. As

$$
m_{0}=0, \quad \text { and } \quad\left(\begin{array}{c}
N-1 \\
-1
\end{array}\right)=0,
$$

it is trivial that (2.6) also holds at $n=0$.

In a similar manner,

$$
Q^{\prime}(z)=\sum_{n=0}^{N-1} c_{n}\left(m_{N}-m_{n}\right) z^{m_{N}-m_{n}-1}
$$

is a sum of strictly less that $N+1$ monomials. We apply the inductive hypothesis to $Q^{\prime}$, and get the inequality

$$
\left|c_{n}\right|\left(m_{N}-m_{n}\right) \leq\left(\begin{array}{c}
N-1 \\
N-1-n
\end{array}\right) \mathfrak{M}\left(Q^{\prime}\right) \leq m_{N}\left(\begin{array}{c}
N-1 \\
n
\end{array}\right) \mathfrak{M}(Q)
$$

for each $n=0,1,2, \ldots, N-1$. In this case we have

$$
\left(m_{N}-m_{N}\right)=0, \quad \text { and } \quad\left(\begin{array}{c}
N-1 \\
N
\end{array}\right)=0,
$$

and therefore (2.7) also holds at $n=N$.

To complete the proof we use the identity (2.4), and we apply the inequality (2.6), and the inequality (2.7). In this way we obtain the bound

$$
\begin{aligned}
\left|c_{n}\right| m_{N} & =\left|c_{n}\right| m_{n}+\left|c_{n}\right|\left(m_{N}-m_{n}\right) \\
& \leq m_{N}\left(\begin{array}{c}
N-1 \\
n-1
\end{array}\right) \mathfrak{M}(P)+m_{N}\left(\begin{array}{c}
N-1 \\
n
\end{array}\right) \mathfrak{M}(P) \\
& =m_{N}\left(\begin{array}{c}
N \\
n
\end{array}\right) \mathfrak{M}(P) .
\end{aligned}
$$

This verifies (1.6).

\section{ARChimEdEAN ORDERINGS IN THE GROUP $\mathbb{Z}^{M}$}

In this section we consider $\mathbb{Z}^{M}$ as an ordered group. To avoid degenerate situations, we assume throughout this section that $2 \leq M$.

Let $\boldsymbol{\alpha}$ belong to $\mathbb{R}^{M}$, and let $\varphi_{\boldsymbol{\alpha}}: \mathbb{Z}^{M} \rightarrow \mathbb{R}$ be the homomorphism defined by (1.13). We assume that the coordinates $\alpha_{1}, \alpha_{2}, \ldots, \alpha_{M}$, are $\mathbb{Q}$-linearly independent so that $\varphi_{\boldsymbol{\alpha}}$ is an injective homomorphism. It follows, as in [13, Theorem 8.1.2 (c)], that $\varphi_{\boldsymbol{\alpha}}$ induces an archimedean ordering in the group $\mathbb{Z}^{M}$. That is, if $\boldsymbol{k}$ and $\boldsymbol{\ell}$ are distinct points in $\mathbb{Z}^{M}$ we write $\boldsymbol{k}<\boldsymbol{\ell}$ if and only if

$$
\varphi_{\boldsymbol{\alpha}}(\boldsymbol{k})=\boldsymbol{k}^{T} \boldsymbol{\alpha}<\varphi_{\boldsymbol{\alpha}}(\ell)=\ell^{T} \boldsymbol{\alpha}
$$


in $\mathbb{R}$. Therefore $\left(\mathbb{Z}^{M},<\right)$ is an ordered group, and the order is archimedean. If $\mathfrak{S} \subseteq \mathbb{Z}^{M}$ is a nonempty, finite subset of cardinality $N+1$, then the elements of $\mathfrak{S}$ can be indexed so that

$$
\mathfrak{S}=\left\{\boldsymbol{k}_{0}, \boldsymbol{k}_{1}, \boldsymbol{k}_{2}, \ldots, \boldsymbol{k}_{N}\right\}
$$

and

$$
\boldsymbol{k}_{0}^{T} \boldsymbol{\alpha}<\boldsymbol{k}_{1}^{T} \boldsymbol{\alpha}<\boldsymbol{k}_{2}^{T} \boldsymbol{\alpha}<\cdots<\boldsymbol{k}_{N}^{T} \boldsymbol{\alpha} .
$$

A more general discussion of ordered groups is given in [13, Chapter 8]. Here we require only the indexing (3.1) that is induced in the finite subset $\mathfrak{S}$ by the injective homomorphism $\varphi_{\boldsymbol{\alpha}}$.

If $\boldsymbol{b}=\left(b_{m}\right)$ is a (column) vector in $\mathbb{Z}^{M}$, we define the norm

$$
\|\boldsymbol{b}\|_{\infty}=\max \left\{\left|b_{m}\right|: 1 \leq m \leq M\right\} .
$$

And if $\mathfrak{S} \subseteq \mathbb{Z}^{M}$ is a nonempty, finite subset we write

$$
\|\mathfrak{S}\|_{\infty}=\max \left\{\|\boldsymbol{k}\|_{\infty}: \boldsymbol{k} \in \mathfrak{S}\right\} .
$$

Following Boyd 2, we define the function

$$
\nu: \mathbb{Z}^{M} \backslash\{\mathbf{0}\} \rightarrow\{1,2,3, \ldots\}
$$

by

$$
\nu(\boldsymbol{a})=\min \left\{\|\boldsymbol{b}\|_{\infty}: \boldsymbol{b} \in \mathbb{Z}^{M}, \boldsymbol{b} \neq \mathbf{0}, \text { and } \boldsymbol{b}^{T} \boldsymbol{a}=0\right\} .
$$

It is known (see [2]) that the function $\boldsymbol{a} \mapsto \nu(\boldsymbol{a})$ is unbounded, and a stronger conclusion follows from our Lemma 3.2. Moreover, if $\nu(\boldsymbol{a})$ is sufficiently large, then the map $\boldsymbol{k} \mapsto \boldsymbol{k}^{T} \boldsymbol{a}$ restricted to points $\boldsymbol{k}$ in the finite subset $\mathfrak{S}$ takes distinct integer values, and therefore induces an ordering in $\mathfrak{S}$. This follows immediately from the triangle inequality for the norm (3.3), and was noted in [2]. As this result will be important in our proof of Theorem 1.2. we prove it here as a separate lemma.

Lemma 3.1. [D. BoYD] Let $\mathfrak{S} \subseteq \mathbb{Z}^{M}$ be a nonempty, finite subset with cardinality $|\mathfrak{S}|=N+1$, and let $\boldsymbol{a} \neq \mathbf{0}$ be a point in $\mathbb{Z}^{M}$ such that

$$
2\|\mathfrak{S}\|_{\infty}<\nu(\boldsymbol{a}) .
$$

Then

$$
\left\{\boldsymbol{k}^{T} \boldsymbol{a}: \boldsymbol{k} \in \mathfrak{S}\right\}
$$

is a collection of $N+1$ distinct integers.

Proof. If $N=0$ the result is trivial. Assume that $1 \leq N$, and let $\boldsymbol{k}$ and $\boldsymbol{\ell}$ be distinct points in $\mathfrak{S}$. If

$$
\boldsymbol{k}^{T} \boldsymbol{a}=\ell^{T} \boldsymbol{a},
$$

then

$$
(\boldsymbol{k}-\ell)^{T} \boldsymbol{a}=\mathbf{0} .
$$

It follows that

$$
\nu(\boldsymbol{a}) \leq\|\boldsymbol{k}-\boldsymbol{\ell}\|_{\infty} \leq\|\boldsymbol{k}\|_{\infty}+\|\boldsymbol{\ell}\|_{\infty} \leq 2\|\mathfrak{S}\|_{\infty},
$$

and this contradicts the hypothesis (3.5). We conclude that (3.6) contains $N+1$ distinct integers. 
Let $\varphi_{\boldsymbol{\alpha}}: \mathbb{Z}^{M} \rightarrow \mathbb{R}$ be an injective homomorphism, and let $\mathfrak{S} \subseteq \mathbb{Z}^{M}$ be a nonempty, finite subset of cardinality $N+1$. We assume that the elements of $\mathfrak{S}$ are indexed so that both (3.1) and (3.2) hold. If $\boldsymbol{a} \neq \mathbf{0}$ in $\mathbb{Z}^{M}$ satisfies (3.5), then it may happen that the indexing (3.1) also satisfies the system of inequalities

$$
\boldsymbol{k}_{0}^{T} \boldsymbol{a}<\boldsymbol{k}_{1}^{T} \boldsymbol{a}<\boldsymbol{k}_{2}^{T} \boldsymbol{a}<\cdots<\boldsymbol{k}_{N}^{T} \boldsymbol{a} .
$$

We write $\mathcal{B}(\boldsymbol{\alpha}, \mathfrak{S})$ for the collection of such lattice points $\boldsymbol{a}$. That is, we define

$$
\begin{aligned}
\mathcal{B}(\boldsymbol{\alpha}, \mathfrak{S})=\left\{\boldsymbol{a} \in \mathbb{Z}^{M}: 2\|\mathfrak{S}\|_{\infty}<\nu(\boldsymbol{a})\right. \\
\left.\quad \text { and } \boldsymbol{k}_{0}^{T} \boldsymbol{a}<\boldsymbol{k}_{1}^{T} \boldsymbol{a}<\boldsymbol{k}_{2}^{T} \boldsymbol{a}<\cdots<\boldsymbol{k}_{N}^{T} \boldsymbol{a}\right\} .
\end{aligned}
$$

The following lemma establishes a crucial property of $\mathcal{B}(\boldsymbol{\alpha}, \mathfrak{S})$.

Lemma 3.2. Let the subset $\mathcal{B}(\boldsymbol{\alpha}, \mathfrak{S})$ be defined by (3.7). Then $\mathcal{B}(\boldsymbol{\alpha}, \mathfrak{S})$ is an infinite set, and the function $\nu$ restricted to $\mathcal{B}(\boldsymbol{\alpha}, \mathfrak{S})$, is unbounded on $\mathcal{B}(\boldsymbol{\alpha}, \mathfrak{S})$.

Proof. By hypothesis

$$
\eta=\eta(\boldsymbol{\alpha}, \mathfrak{S})=\min \left\{\boldsymbol{k}_{n}^{T} \boldsymbol{\alpha}-\boldsymbol{k}_{n-1}^{T} \boldsymbol{\alpha}: 1 \leq n \leq N\right\}
$$

is a positive constant that depends on $\boldsymbol{\alpha}$ and $\mathfrak{S}$.

By Dirichlet's theorem in Diophantine approximation (see [3] or [15]), for each positive integer $Q$ there exists an integer $q$ such that $1 \leq q \leq Q$, and

$$
\max \left\{\left\|q \alpha_{m}\right\|: m=1,2, \ldots, M\right\} \leq(Q+1)^{-\frac{1}{M}} \leq(q+1)^{-\frac{1}{M}},
$$

where \|\| on the left of (3.9) is the distance to the nearest integer function. Let $\mathcal{Q}$ be the collection of positive integers $q$ such that

$$
\max \left\{\left\|q \alpha_{m}\right\|: m=1,2, \ldots, M\right\} \leq(q+1)^{-\frac{1}{M}} .
$$

Because $2 \leq M$, at least one of the coordinates $\alpha_{m}$ is irrational, and it follows from (3.9) that $\mathcal{Q}$ is an infinite set.

For each positive integer $q$ in $\mathcal{Q}$, we select integers $b_{1 q}, b_{2 q}, \ldots, b_{M q}$, so that

$$
\left\|q \alpha_{m}\right\|=\left|q \alpha_{m}-b_{m q}\right|, \quad \text { for } m=1,2, \ldots, M .
$$

Then (3.10) can be written as

$$
\max \left\{\left|q \alpha_{m}-b_{m q}\right|: m=1,2, \ldots, M\right\} \leq(q+1)^{-\frac{1}{M}} .
$$

Let $\boldsymbol{b}_{q}=\left(b_{m q}\right)$ be the corresponding lattice point in $\mathbb{Z}^{M}$, so that $q \mapsto \boldsymbol{b}_{q}$ is a map from $\mathcal{Q}$ into $\mathbb{Z}^{M}$. It follows using (3.8) and (3.12), that for each index $n$ we have

$$
\begin{aligned}
q \eta & \leq q \boldsymbol{k}_{n}^{T} \boldsymbol{\alpha}-q \boldsymbol{k}_{n-1}^{T} \boldsymbol{\alpha} \\
& =\boldsymbol{k}_{n}^{T} \boldsymbol{b}_{q}-\boldsymbol{k}_{n-1}^{T} \boldsymbol{b}_{q}+\left(\boldsymbol{k}_{n}-\boldsymbol{k}_{n-1}\right)^{T}\left(q \boldsymbol{\alpha}-\boldsymbol{b}_{q}\right) \\
& \leq \boldsymbol{k}_{n}^{T} \boldsymbol{b}_{q}-\boldsymbol{k}_{n-1}^{T} \boldsymbol{b}_{q}+2\|\mathfrak{S}\|_{\infty}\left(\sum_{m=1}^{M}\left|q \alpha_{m}-b_{m q}\right|\right) \\
& \leq \boldsymbol{k}_{n}^{T} \boldsymbol{b}_{q}-\boldsymbol{k}_{n-1}^{T} \boldsymbol{b}_{q}+2\|\mathfrak{S}\|_{\infty} M(q+1)^{-\frac{1}{M}} .
\end{aligned}
$$

Therefore for each sufficiently large integer $q$ in $\mathcal{Q}$, the lattice point $\boldsymbol{b}_{q}$ satisfies the system of inequalities

$$
\boldsymbol{k}_{0}^{T} \boldsymbol{b}_{q}<\boldsymbol{k}_{1}^{T} \boldsymbol{b}_{q}<\boldsymbol{k}_{2}^{T} \boldsymbol{b}_{q}<\cdots<\boldsymbol{k}_{N}^{T} \boldsymbol{b}_{q}
$$

We conclude that for a sufficiently large integer $L$ we have

$$
\left\{\boldsymbol{b}_{q}: L \leq q \text { and } q \in \mathcal{Q}\right\} \subseteq \mathcal{B}(\boldsymbol{\alpha}, \mathfrak{S}) .
$$


This shows that $\mathcal{B}(\boldsymbol{\alpha}, \mathfrak{S})$ is an infinite set.

To complete the proof we will show that the function $\nu$ is unbounded on the infinite collection of lattice points

$$
\left\{\boldsymbol{b}_{q}: L \leq q \text { and } q \in \mathcal{Q}\right\} .
$$

If $\nu$ is bounded on (3.14), then there exists a positive integer $B$ such that

$$
\nu\left(\boldsymbol{b}_{q}\right) \leq B
$$

for all points $\boldsymbol{b}_{q}$ in the set (3.14). Let $\mathcal{C}_{B}$ be the finite set

$$
\mathcal{C}_{B}=\left\{\boldsymbol{c} \in \mathbb{Z}^{M}: 1 \leq\|\boldsymbol{c}\|_{\infty} \leq B\right\} .
$$

Because $\alpha_{1}, \alpha_{2}, \ldots, \alpha_{M}$, are $\mathbb{Q}$-linearly independent, and $\mathcal{C}_{B}$ is a finite set of nonzero lattice points, we have

$$
0<\delta_{B}=\min \left\{\left|\sum_{m=1}^{M} c_{m} \alpha_{m}\right|: c \in \mathcal{C}_{B}\right\} .
$$

By our assumption (3.15), for each point $\boldsymbol{b}_{q}$ in (3.14) there exists a point $\boldsymbol{c}_{q}=\left(c_{m q}\right)$ in $\mathcal{C}_{B}$, such that

$$
\boldsymbol{c}_{q}^{T} \boldsymbol{b}_{q}=\sum_{m=1}^{M} c_{m q} b_{m q}=0
$$

Using (3.12) and (3.17), we find that

$$
\begin{aligned}
q \delta_{B} & \leq q\left|\sum_{m=1}^{M} c_{m q} \alpha_{m}\right| \\
& =\left|\sum_{m=1}^{M} c_{m q}\left(q \alpha_{m}-b_{m q}\right)\right| \\
& \leq\left(\sum_{m=1}^{M}\left|c_{m q}\right|\right) \max \left\{\left|q \alpha_{m}-b_{m q}\right|: m=1,2, \ldots, M\right\} \\
& \leq M B(q+1)^{-\frac{1}{M}} .
\end{aligned}
$$

But (3.18) is impossible when $q$ is sufficiently large, and the contradiction implies that the assumption (3.15) is false. We have shown that $\nu$ is unbounded on the set (3.14). In view of (3.13), the function $\nu$ is unbounded on $\mathcal{B}(\boldsymbol{\alpha}, \mathfrak{S})$.

\section{Proof of Theorem 1.2}

If $M=1$ then the inequality (1.16) follows from Corollary 1.1. Therefore we assume that $2 \leq M$.

Let $\varphi_{\boldsymbol{\alpha}}: \mathbb{Z}^{M} \rightarrow \mathbb{R}$ be an injective homomorphism, and let the set $\mathfrak{S}$ be indexed so that (1.14) and (1.15) hold. It follows from Lemma 3.2 that the collection of lattice points $\mathcal{B}(\boldsymbol{\alpha}, \mathfrak{S})$ defined by (3.7), is an infinite set, and the function $\nu$ defined by (3.4) is unbounded on $\mathcal{B}(\boldsymbol{\alpha}, \mathfrak{S})$.

Let $\boldsymbol{a}$ be a lattice point in $\mathcal{B}(\boldsymbol{\alpha}, \mathfrak{S})$. If $F:(\mathbb{R} / \mathbb{Z})^{M} \rightarrow \mathbb{C}$ is given by (1.12), we define an associated trigonometric polynomial $F_{\boldsymbol{a}}: \mathbb{R} / \mathbb{Z} \rightarrow \mathbb{C}$ in one variable by

$$
F_{\boldsymbol{a}}(t)=\sum_{\boldsymbol{k} \in \mathfrak{S}} \widehat{F}(\boldsymbol{k}) e\left(\boldsymbol{k}^{T} \boldsymbol{a} t\right)=\sum_{n=0}^{N} \widehat{F}\left(\boldsymbol{k}_{n}\right) e\left(\boldsymbol{k}_{n}^{T} \boldsymbol{a} t\right),
$$


where the equality on the right of (4.1) uses the indexing (1.14) induced by $\varphi_{\boldsymbol{\alpha}}$. The hypothesis (1.15) implies that the integer exponents on the right of (4.1) satisfy the system of inequalities

$$
\boldsymbol{k}_{0}^{T} \boldsymbol{a}<\boldsymbol{k}_{1}^{T} \boldsymbol{a}<\boldsymbol{k}_{2}^{T} \boldsymbol{a}<\cdots<\boldsymbol{k}_{N}^{T} \boldsymbol{a} .
$$

Then it follows from (1.8), (4.1), and (4.2), that

$$
\left|\widehat{F}\left(\boldsymbol{k}_{n}\right)\right| \leq\left(\begin{array}{c}
N \\
n
\end{array}\right) \mathfrak{M}\left(F_{\boldsymbol{a}}\right), \quad \text { for each } n=0,1,2, \ldots, N .
$$

We have proved that the system of inequalities (4.3) holds for each lattice point $\boldsymbol{a}$ in $\mathcal{B}(\boldsymbol{\alpha}, \mathfrak{S})$.

To complete the proof we appeal to an inequality of Boyd [2, Lemma 2], which asserts that if $\boldsymbol{b}$ is a parameter in $\mathbb{Z}^{M}$ then

$$
\limsup _{\nu(\boldsymbol{b}) \rightarrow \infty} \mathfrak{M}\left(F_{\boldsymbol{b}}\right) \leq \mathfrak{M}(F)
$$

More precisely, if $\boldsymbol{b}_{1}, \boldsymbol{b}_{2}, \boldsymbol{b}_{3}, \ldots$, is a sequence of points in $\mathbb{Z}^{M}$ such that

$$
\lim _{j \rightarrow \infty} \nu\left(\boldsymbol{b}_{j}\right)=\infty
$$

then

$$
\limsup _{j \rightarrow \infty} \mathfrak{M}\left(F_{\boldsymbol{b}_{j}}\right) \leq \mathfrak{M}(F) .
$$

Because $\nu$ is unbounded on $\mathcal{B}(\boldsymbol{\alpha}, \mathfrak{S})$, there exists a sequence $\boldsymbol{b}_{1}, \boldsymbol{b}_{2}, \boldsymbol{b}_{3}, \ldots$, contained in $\mathcal{B}(\boldsymbol{\alpha}, \mathfrak{S})$ that satisfies (4.5). Hence the sequence $\boldsymbol{b}_{1}, \boldsymbol{b}_{2}, \boldsymbol{b}_{3}, \ldots$, in $\mathcal{B}(\boldsymbol{\alpha}, \mathfrak{S})$ also satisfies (4.6). From (4.3) we have

$$
\left|\widehat{F}\left(\boldsymbol{k}_{n}\right)\right| \leq\left(\begin{array}{c}
N \\
n
\end{array}\right) \mathfrak{M}\left(F_{\boldsymbol{b}_{j}}\right),
$$

for each $n=0,1,2, \ldots, N$, and for each $j=1,2,3, \ldots$ The inequality (1.16) plainly follows from (4.6) and (4.7). This completes the proof of Theorem 1.2

Boyd conjectured in 2 that (4.4) could be improved to

$$
\lim _{\nu(\boldsymbol{b}) \rightarrow \infty} \mathfrak{M}\left(F_{\boldsymbol{b}}\right)=\mathfrak{M}(F) .
$$

The proposed identity (4.8) was later verified by Lawton [8] (see also [4] and [7]). Here we have used Boyd's inequality (4.4) because it is simpler to prove than (4.8), and the more precise result (4.8) does not effect the inequality (1.16).

\section{REFERENCES}

[1] E. Bombieri and W. Gubler, Heights in Diophantine Geometry, Cambridge U. Press, New York, 2006.

[2] D. W. Boyd, Kronecker's Theorem and Lehmer's Problem for Polynomials in Several Variables, Journal of Number Theory 13, (1981), 116-121.

[3] J. W. S. Cassels, An Introduction to Diophantine Approximation, Cambridge U. Press, London, 1965.

[4] E. Dobrowolski, A note on Lawton's theorem, Canad. Math. Bull. 60, no. 3, (2017), 484-489.

[5] E. Dobrowolski and C. Smyth, Mahler measures of polynomials that are sums of a bounded number of monomials, International Journal of Number Theory, 13, No. 6 (2017), 1603-1610.

[6] G. Everest and T. Ward, Heights of Polynomials and Entropy in Algebraic Dynamics, Springer-Verlag, London, 1999.

[7] Z. Issa and M. Lalín, A Generalization of a Theorem of Boyd and Lawton, Canad. Math. Bull. 56, no. 4, (2013), 759-768. 
[8] W. M. Lawton, A Problem of Boyd concerning Geometric Means of Polynomials, Journal of Number Theory, 16 (1983), 356-362.

[9] K. Mahler, An application of Jensen's formula to polynomials, Mathematika, 7 (1960), 98-100.

[10] K. Mahler, On the zeros of the derivative of a polynomial, Proceedings of the Royal Society, Ser. A, (London) Vol. 264, (1961), 145-154.

[11] K. Mahler, Some inequalities for polynomials in several variables, Journal London Math. Soc., 37 (1962), 341-344.

[12] I. E. Pritsker, Mahler's measure, and multipliers, in Number Theory and Polynomials, ed. J. McKee and C. Smyth, London Math. Soc. Lecture Notes Series, 352, 255-276.

[13] W. Rudin, Fourier Analysis on Groups Interscience Pub., New York, (1962).

[14] K. Schmidt, Dynamical Systems of Algebraic Origin, Birkhäuser Verlag, Basel, 1995.

[15] W. M. Schmidt, Diophantine Approximation, Lecture Notes in Mathematics 785, SpringerVerlag, Berlin, 1980.

[16] C. Smyth, The Mahler measure of algebraic numbers: a survey, in Number Theory and Polynomials, ed. J. McKee and C. Smyth, London Math. Soc. Lecture Notes Series, 352, $322-349$.

Department of Mathematics, University of Oregon, Eugene, Oregon 97402 USA, Max Planck Institute for Mathematics, Vivatsgasse 7, 53111 Bonn, Germany

E-mail address: akhtari@uoregon.edu

Department of Mathematics, University of Texas, Austin, Texas 78712 USA

E-mail address: vaaler@math.utexas.edu 